\title{
Sitting position improves consciousness level in patients with cerebral disorders
}

\author{
Takashi Moriki $^{1}$, Takeshi Nakamura ${ }^{1^{*}}$, Daisuke Kojima ${ }^{1}$, Hiroyoshi Fujiwara ${ }^{2}$, Fumihiro Tajima ${ }^{1}$ \\ ${ }^{1}$ Department of Rehabilitation Medicine, Wakayama Medical University, Wakayama, Japan; \\ *Corresponding Author: take-n@wakayama-med.ac.jp \\ ${ }^{2}$ Department of Orthopedics, Graduate School of Medical Science, Kyoto Prefectural University of Medicine, Kyoto, Japan
}

Received 2 May 2013; revised 3 June 2013; accepted 12 June 2013

Copyright ( 2013 Takashi Moriki et al. This is an open access article distributed under the Creative Commons Attribution License, which permits unrestricted use, distribution, and reproduction in any medium, provided the original work is properly cited.

\begin{abstract}
Cerebral disorders are often associated with disturbance of consciousness. Since the latter could alter the prognosis of physical function, early improvement of consciousness level is important. The present study tested the hypothesis that sitting position improves disturbances of consciousness in patients with cerebral disorders. The subjects were 17 patients with cerebral disorders and disturbances of consciousness. The consciousness level was evaluated in supine position and following a change to sitting position. The consciousness level was evaluated by the Glasgow Coma Scale (GCS). The total, eye opening, and motor GCS scores were significantly higher in the sitting position compared with the supine position. In conclusions, changing from supine to sitting position improves consciousness level in patients with cerebral disorders.
\end{abstract}

Keywords: Consciousness Disturbance; Glasgow Coma Scale; Sitting Position

\section{INTRODUCTION}

The major clinical features of cerebral disorders (e.g., cerebrovascular accident, brain injury, brain tumor and hypoxic encephalopathy) are disturbance of consciousness, hemiplegia, sensory disturbance and higher cerebral dysfunction [1]. Disturbance of consciousness can indirectly affect prognosis of physical dysfunction. Therefore, it is important to improve as soon as possible the consciousness level of patients with cerebral disorders.

The reticular activating system (RAS) is primarily involved in the state of arousal and wakefulness and nor- mally responds to all sensory stimuli, including pain, pressure, touch, temperature, proprioception, vision, and hearing [2]. Intense multisensory stimulation in patients with disturbances of consciousness should stimulate the RAS and improve arousability. For example, clinical evidence indicates that sensory stimulation (e.g., intermittent bright lights directed into the patient's eyes), sound (clapping two blocks of wood together periodically next to the patient's ear), and sensory stimulation (touching extremities with ice cubes) improve the consciousness level [3].

A change in posture from supine to sitting position adds gravitation stress to the body. Thus, the sitting position might stimulate RAS and improve consciousness level in patients with cerebral disorders. To our knowledge, however, there is no information on the effect of sitting position on disturbance of consciousness. We tested in this study the hypothesis that sitting position improves disturbances of consciousness.

\section{METHODS}

\subsection{Participants}

The study subjects were 17 patients with disturbances of consciousness (mean age, $71.3 \pm 14.0$ years; \pm SD, range, 35 - 90, including 7 with cerebral infarction, 4 with cerebral hemorrhage, and 6 with traumatic brain injury). They consisted of 9 men (70.3 \pm 10.1 years; range, $49-81)$ and 8 women (72.4 \pm 18.1 years; range, 35 - 90). Table 1 summarizes the characteristics of the participating subjects. The study was approved by the ethics review committee of Wakayama Medical University, School of Medicine, and was performed between October 2007 and September 2008. Families of all subjects were informed about the study procedure before signing the consent form in accordance to the guidelines of the Wakayama Medical University. 
Table 1. Patient characteristics and causes of brain injury.

\begin{tabular}{lc}
\hline Age (years), mean \pm SD (range) & $71.3 \pm 13.0(35-90)$ \\
\hline Time since disease (days) & $30 \pm 18.6(6-62)$ \\
Gender, $n(\%)$ & $9(53)$ \\
Males & $8(47)$ \\
Females & \\
Cause of brain injury, n (\%) & $7(41)$ \\
Cerebral infarction & $4(24)$ \\
Cerebral hemorrhage & $6(35)$ \\
Traumatic brain injury &
\end{tabular}

\subsection{Measurements}

The level of consciousness was evaluated using the Glasgow Coma Scale (GCS) developed by Teasdale and Jannett [4]. GCS is used as an assessment tool to measure the level of consciousness (eye opening in response to stimuli, verbal response to stimuli, motor response to stimuli). This is a highly reliable and valid measure that has been validated in many studies and is therefore used widely in clinical practice [5,6]. The GCS score varies from 3 to 15 points, and a low score indicates a low consciousness level. Two researchers were involved in the conduct of the study protocol; one who assisted the patient to change body posture and another person who evaluated the level of consciousness. To avoid interrater differences, only one researcher evaluated the consciousness level in all 17 patients.

\subsection{Study Protocol}

The level of consciousness was evaluated while the subject was in comfortable supine and sitting positions. First, the consciousness level was evaluated in the supine position. Then, the subject changed posture from supine to sitting position, and the consciousness level was evaluated immediately after such change. Minimum assistance was required to help the patient change posture from supine to sitting in all subjects.

\subsection{Statistical Analysis}

All data were expressed as median (IQR). The Wilcoxon's matched-pairs signed-rank test was used for comparison of the GCS scores of supine and sitting positions. A $P$ value less than 0.05 denoted the presence of a statistically significant difference.

\section{RESULTS}

The median total GCS score in the supine position was 7 (IQR, 6 to 11), with eye opening GCS score of 3 (IQR, 2 to 3), verbal response GCS score of 1 (IQR, 1 to 2), and motor response of 4 (IQR, 3 to 5). The median total
GCS score in the sitting position was 11 (IQR, 8 to 14), with eye opening GCS score of 4 (IQR, 4 to 4 ), verbal response of 2 (IQR, 1 to 4), and motor response GCS score of 5 (IQR, 3 to 6 ).

Sitting position significantly improved the median total GCS score $(P<0.005)$ (Figure 1(a)), the median eye opening GCS score $(P<0.005)$ (Figure 1(b)), and the median motor response GCS score $(P<0.05)$ (Figure 1(d)), compared with supine position. However, sitting from supine position did not improve the verbal response GCS score (Figure 1(c)).

\section{DISCUSSION}

The results of the present study confirmed our hypothesis that sitting position improves the GCS score relative to the supine position. Based on the results, we conclude that stimulation by changing from supine position to sitting position seems to have a positive influence on the level of consciousness in patients with disturbance of consciousness.

The RAS is a group of neurons that transcends through the brainstem and thalamus, and connects the cerebral cortex with the spinal cord. The RAS controls wakefulness, cortical alertness, ability of concentrate, perception and modulation of sensory information [6]. RAS is sensitive to alterations in pain, medications and sensory deprivation. Sensory stimulation triggers impulses that

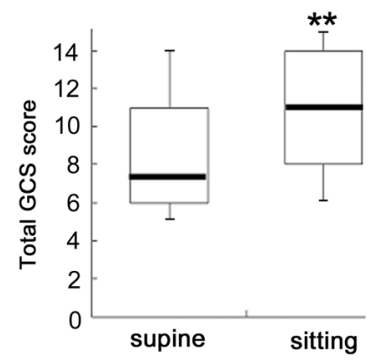

(a)

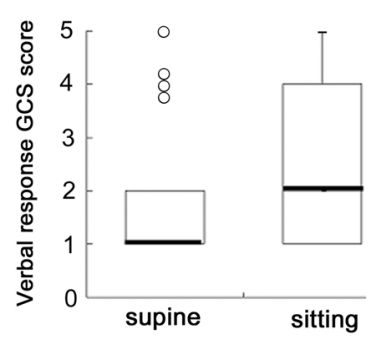

(c)

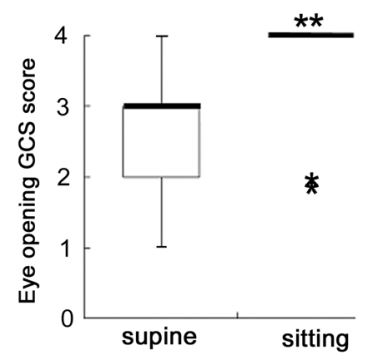

(b)

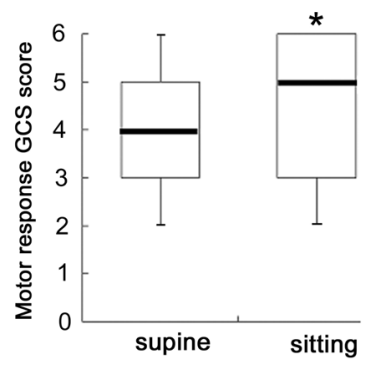

(d)
Figure 1. The median values of the Glasgow Coma Scale (GCS) scores measured in supine and sitting positions, including total GCS score (a), eye opening GCS score (b), verbal response GCS score (c) and motor response GCS score (d) of 17 patients. ${ }^{*} P<0.05,{ }^{* *} P<0.005$, compared with the supine GCS score (by Wilcoxon matched-pairs signed-rank test). 
are transmitted to the RAS, which ultimately lead to activation of the cerebral cortex. The results of the present study suggest that gravitation stress induced by sitting position stimulated the RAS, resulting in improvement of the GCS score.

In present study, the eye opening and motor response GCS scores significantly improved at sitting compared with supine position. However, the change in body posture did not affect the verbal response GCS score. Sixteen of the 17 patients who participated in this study had of bilateral cerebral cortical damage. Damage of the cerebral cortex influences cognitive function. Therefore, most patients of our study could have verbal cognitive dysfunction. We speculate that cognitive dysfunction could have a negative influence on the verbal response GCS score.

The present study has certain limitations. The study did not evaluate the effect of repetition of the sitting position on the improvement of disturbances of consciousness. Further studies are needed to evaluate the level of consciousness in the sitting position on several days to determine the effect of repetitive change in body posture.

We have demonstrated in the present study that the sitting position effectively improve consciousness level in patients with cerebral disorders. The results suggest that further improvement in consciousness level could probably be achieved on standing position since the latter position is more stressful posture than sitting position. Physical therapists do not need special techniques to change body posture of patients with cerebral disorders, since this can be achieved at bedside. The present results suggest that a posture change is easy and beneficial rehabilitation program for patients with disturbances of consciousness.

\section{CONCLUSION}

The present study demonstrated that sitting position improved the GCS score in patients with cerebral disorders and disturbances of consciousness, suggesting the effectiveness of the sitting position in improving consciousness level.

\section{ACKNOWLEDGEMENTS}

We thank Takeshi Harada, Takamasa Hashizaki and Tokio Kinoshita for the clinical assistance. We also thank Dr. Faiq G Issa for the careful reading and editing of the manuscript.

\section{REFERENCES}

[1] Scherer, P. (1986) Assessment the logic of coma. American Journal of Nursing, 86, 542-549.

[2] Spielman, G. (1981) Coma: A clinical review. Heart and Lung, 10, 700-707.

[3] DeYoung, S. and Grass, R.B. (1987) Coma recovery program. Rehabilitation Nursing, 12, 121-124. doi:10.1002/j.2048-7940.1987.tb00573.x

[4] Teasdale, G. and Jennett, B. (1974) Assessment of coma and impaired consciousness. A practical scale. Lancet, 13, 81-84. doi:10.1016/S0140-6736(74)91639-0

[5] Oh, H. and Seo, W. (2003) Sensory stimulation programme to improve recovery in comatose patients. Journal of Clinical Nursing, 12, 394-404. doi:10.1046/j.1365-2702.2003.00750.x

[6] Sosnowski, C. and Ustik, M. (1994) Early intervention: Coma stimulation in the intensive care unit. Journal of Neuroscience Nursing, 26, 336-341. doi:10.1097/01376517-199412000-00005 